(C) Cambridge University Press 2014. This is an Open Access article, distributed under the terms of the Creative Commons Attribution licence (http://creativecommons.org/ licenses/by/3.0/), which permits unrestricted re-use, distribution, and reproduction in any medium, provided the original work is properly cited.

\title{
Pea aphid biotype performance on diverse Medicago host genotypes indicates highly specific virulence and resistance functions
}

\author{
S. Kanvil, G. Powell and C. Turnbull* \\ Department of Life Sciences, Imperial College London, \\ London SW7 2AZ, UK
}

\begin{abstract}
Aphid-plant interactions depend on genotypes of both organisms, which determine the two-way molecular exchange that leads to compatible or incompatible outcomes. The underlying genes are mostly unknown, making it difficult to predict likelihood of aphid success or host resistance, and hampering crop genetic improvement. Here we screened eight pea aphid clonal genotypes collected from diverse legume hosts, on a species-wide panel of Medicago truncatula (Mt) genotypes. Aphid virulence was measured by survival, fecundity and growth rate, together with scores for chlorosis and necrosis as host response indicators. Outcomes were highly dependent on the specific aphid-host genotype combinations. Only one Mt line was fully resistant against all clones. Aphid-induced host chlorosis and necrosis varied greatly, but correlated with resistance only in a few combinations. Bi-clustering analysis indicated that all aphid clones could be distinguished by their performance profiles across the host genotypes tested, with each clone being genetically differentiated and potentially representing a distinct biotype. Clones originating from Medicago sativa ranged from highly virulent to almost completely avirulent on both Medicago species, indicating that some were well adapted, whereas others were most likely migrants. Comparisons of closely related pairs of Australian Mt genotypes differing in aphid resistance revealed no enhanced resistance to European pea aphid clones. Based on the extensive variation in pea aphid adaptation even on unfamiliar hosts, most likely reflecting multiple biotype-specific gene-for-gene interactions, we conclude that robust defences require an arsenal of appropriate resistance genes.
\end{abstract}

Keywords: biotype, chlorosis, diversity, necrosis, pest resistance, virulence

(Accepted 20 May 2014; First published online 23 July 2014)

\section{Introduction}

Major worldwide plant pests include aphids that damage crops directly by phloem feeding and indirectly by transmitting many harmful viruses. Aphids vary in their host range from monophagus to polyphagous. For example, the

*Author for correspondence

Phone: + 442075946437

E-mail: c.turnbull@imperial.ac.uk generalist peach-potato aphid, Myzus persicae, feeds on more than 50 plant families (Schoonhoven et al., 1998), whereas pea aphid (Acyrthosiphon pisum) is specialized on to the legume family, Fabaceae. However, extensive genetic divergence in host adaptation in pea aphid has been reported (Via, 1999). Pea aphid biotypes with distinct adaptations to various sympatric wild and cultivated legumes were first demonstrated by Müller (1962), allowing specialized exploitation of particular host species (Blackman \& Eastop, 2000; Caillaud \& Via, 2000; Ferrari et al., 2006; Peccoud et al., 2009). Biotypes are conventionally defined either as populations that show 
dramatically reduced performance when transferred onto an alternative host (Müller, 1962; Via, 1991; Ferrari et al., 2008; Peccoud et al., 2009), or more broadly as populations within a species that differ in their ability to thrive on a particular plant genotype (Smith, 2005). Although most aphids reproduce predominantly by asexual means, rapid evolution of new host-plant associations is possible, both through mutation and resulting from recombination during sexual reproduction. In agricultural contexts, emergence of better-adapted, resistance-breaking virulent aphid biotypes (Puterka \& Peters, 1989, 1990) presents a major challenge for crop breeding in terms of generating then sustaining durable aphid resistance (Berzonsky et al., 2003; Smith et al., 2004; Kim et al., 2008).

Variation in aphid performance on different host species and between genotypes of the same species is related to both host plant and aphid genotype. One class of resistance involves specific 'gene-for-gene' recognition mechanisms associated with resistance $(R)$ genes in plants and cognate avirulence (Avr) genes in aphids (Kaloshian, 2004). In this model, interaction between $R$ gene and Avr gene products typically leads to incompatibility between pest and the host plant. To date, only a few plant $R$-genes have been isolated that confer resistance against aphids. These include $M i-1.2$ in tomato which is effective against potato aphid (Macrosiphum euphorbiae; Nombela et al., 2003) and Vat in melon (Cucumis melo) which provides resistance to cotton-melon aphid (Aphis gossypii; Klingler et al., 1998). However, none of the cognate aphid Avr gene products, generally referred to as effectors, has yet been conclusively identified, hampering elucidation of the molecular basis of host adaptation.

Screening of Medicago truncatula (Mt) germplasm has identified accessions carrying resistance to a range of aphid species (Crawford et al., 1989), including three independent resistance loci, TTR, $A K R$ and $A P R$, that confer resistance to spotted alfalfa aphid (SAA; Therioaphis trifolii), blue green aphid (BGA; Acyrthosiphon kondoi) and pea aphid, respectively (Gao et al., 2008). Although the underlying genes have not yet been isolated, resistance loci have been introgressed into susceptible cultivars (Lake, 1993a, b; Hill, 2000). Three resultant BGA-resistant cultivars and their corresponding susceptible recurrent backcross parents each share substantial components of their genomes (Crawford et al., 1989; Hill, 2000), providing opportunities to compare pairs of closely related resistant and susceptible genotypes (Jester-Jemalong A17, Mogul-Borung and Caliph-Cyprus).

Mapping studies and allelism experiments suggest that Mogul has the same BGA resistance gene $(A K R)$ as Jester, but may possess different $A K R$ alleles at this locus (Klingler et al., 2005). Resistance to BGA in Caliph is likely to derive from the same source as in Jester (Lake, 1993b; Hill, 2000). However, $A K R$ does not appear to confer resistance to pea aphid in these cultivars (Gao et al., 2008). Instead pea aphid resistance, at least in Jester, is associated with $A P R$, which could be tightly linked to $A K R$ and/or TTR (Gao et al., 2007; Guo et al., 2009, 2012).

In gene-for-gene interactions between pathogens and plant hosts, incompatibility often leads to rapid localized cell death, known as the hypersensitive response (HR), that is especially effective at isolating and minimizing spread of biotrophic pathogens (Garcia-Brugger et al., 2006). An HR-like response may also contribute to aphid resistance (Boyko et al., 2006; Chen, 2008). In Mt, the Acyrthosiphon-induced necrosis (AIN) gene mediates induction of HR in response to both pea aphid and BGA but interestingly confers resistance only to BGA
(Klingler et al., 2009). Likewise, the Aphid-induced lesions (AIL) locus is responsible for HR phenotypes in Mt genotype Jemalong A17 (hereafter called A17) but is not necessary for resistance to pea aphid clone PS01, which instead maps to independent quantitative trait loci such as Resistance to A. pisum 1 (RAP1) (Klingler et al., 2007; Stewart et al., 2009). Similarly, tomato $\mathrm{Mi}$-mediated resistance to potato aphids does not require an HR (Martinez de Ilarduya et al., 2003), but intriguingly, the same gene provides root-knot nematode resistance and in that case is associated with an HR. These differential responses may relate to divergent feeding modes and different feeding sites - shoot versus root. As plant symptoms are not the same in all aphid-plant interactions, the range of responses elicited can represent informative indicators of the mechanisms of resistance.

To achieve durable aphid resistance, it is important to understand the genetic and molecular basis of plant-aphid incompatibility. By screening for plant lines which give broader resistance to multiple aphid genotypes, we aimed to generate pictures both of the extent of variation in virulence across pea aphid populations, and of the species-wide range of resistance present in Mt hosts. Pea aphid clones included in the present study were collected from various host species (table 1) and were predicted to show extensive variation in performance when tested across a wide host range. We assembled a panel of $\mathrm{Mt}$ genotypes (table 2A, B) that includes natural accessions from several geographic locations, together with cultivar pairs mentioned above that differ in reported degree of aphid resistance. These latter genotypes enabled testing of whether the enhanced aphid resistance reported in cvs. Jester, Mogul and Caliph, was effective against pea aphid clones collected in Europe. This survey substantially broadens our knowledge of adaptation, virulence and resistance diversity in the pea aphid-Mt model system, and can enable design of comparative studies on the genetics of aphid virulence and avirulence, leading potentially to future design of robustly resistant crop plants.

\section{Materials and methods}

\section{Aphids}

The eight pea aphid (A. pisum) clones tested were originally collected from field sites in the UK, Ireland or France, and therefore are considered to represent European biotypes. All aphid populations were kept as parthenogenetic colonies (clones) on tic bean (Vicia faba var. minor) at constant $19^{\circ} \mathrm{C}$, relative humidity of $50 \%$ day, $65 \%$ night, under a $16 \mathrm{~h}$ photoperiod. Out of eight clones, four were originally collected from Medicago sativa, whereas the other four were from different legume genera (table 1). Microsatellite loci data, based on the markers reported by Peccoud et al. (2009), on the majority of these clones indicate that they are genetically distinct and fall into different host races (Jean-Christophe Simon, personal communication), the only exception being JF01/29 which was lost from culture prior to genotyping. Endosymbiont screening done in the laboratories of Angela Douglas and Alison Karley at the time of the experiments indicated secondary species in most but not all clones (table 1). To obtain required ages of nymphs, adult aphids were transferred to tic bean seedlings under glass tubes $(2.3 \mathrm{~cm}$ diameter, $5 \mathrm{~cm}$ high) using a fine brush and were allowed to reproduce for $24 \mathrm{~h}$ before being removed. The nymphs produced remained on tic beans under enclosed perforated 


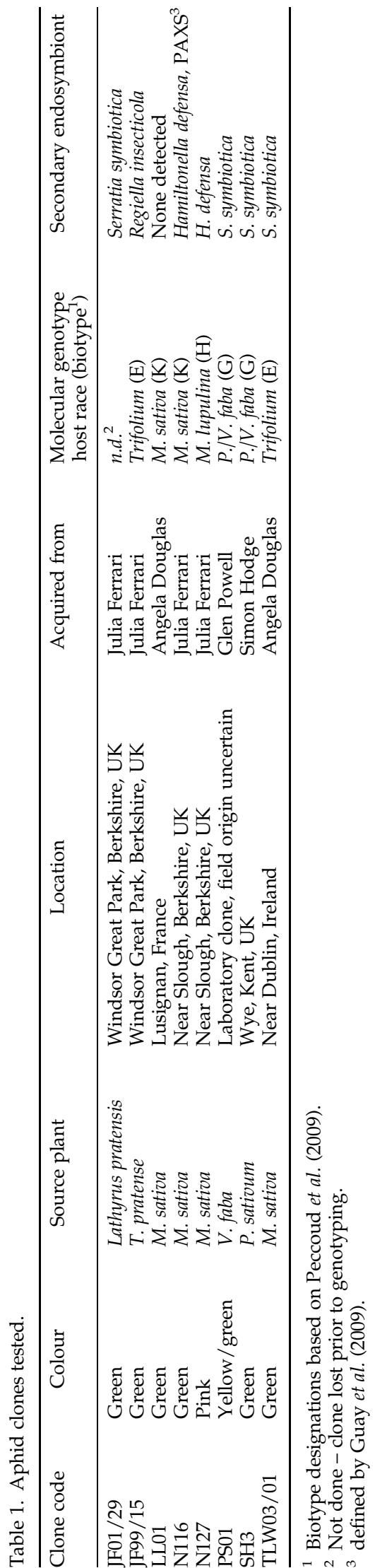

bags for an appropriate number of days before use in experiments.

\section{Plants}

Twenty-three Mt (barrel medic) genotypes were used (table 2). The test panel of germplasm was selected from a wide geographic range, including 15 natural accessions annotated as originating from around the Mediterranean region, i.e. North Africa, Southern Europe and Israel. Three closely related pairs of cultivars bred in Australia were selected along with two progenitor lines (SA10733 and SA1499) used to create the aphid-resistant lines Jester and Caliph (table 2A). These genotypes include substantial northern hemisphere parentage (Lake, 1993b). Many of the Medicago genotypes also feature in the Hapmap core collections (www.medicagohapmap.org/hapmap/germplasm), which were selected to be representative of the species diversity across multiple sites of origin. In addition, $M$. sativa (alfalfa; seed from Bavicchi SPA, Perugia, Italy) was used as several clones were collected from this species. Vicia faba minor (tic bean) was also included as a universal host for all known pea aphid biotypes (Ferrari et al., 2008). Prior to germination, Medicago seeds were scarified with sand paper then imbibed in distilled water for 3-4h at room temperature. After transfer onto damp filter paper in Petri dishes, seeds were incubated overnight in darkness at $4^{\circ} \mathrm{C}$. After returning to room temperature for a further $24 \mathrm{~h}$ in darkness to allow germination, seeds were planted in compost (4:1 Levington's F2S: perlite) in a growth room (temperature $\sim 23 \pm 1^{\circ} \mathrm{C}, 16 \mathrm{~h}$ day length, 50-60\% relative humidity, photosynthetically active radiation $180 \mu \mathrm{molm}^{-2} \mathrm{~s}^{-1}$ from Starcoat $\mathrm{T} 5$ high output (Hungary) fluorescent lamps. Seedlings were transferred to P24 cell trays (Plantpak) at two cotyledon stage approximately 1 week after sowing. Plants were used in experiments at 3 weeks old with 3-5 trifoliate leaves expanded.

\section{Pea aphid and M. truncatula performance}

Plants of Mt genotypes, M. sativa and V. faba were each infested with five (1-2-day-old) nymphs of one of the eight pea aphid clones, and enclosed in a perforated plastic bag. For each aphid clone, ten replicate plants of each host genotype were used. After 8 days, the number of adult aphids surviving and the number of nymphs produced by surviving adults were counted on each plant. Infested plants were also assessed for presence or absence of visual phenotypic symptoms especially chlorosis (pale, yellow or yellow-white leaves, interveinal yellowing of leaves) and necrosis (brownish or whitish spots or patches on the leaves) after 8 days. Data were recorded as presence/absence of symptoms for each plant. As aphid positions on plants were not controlled, no analysis of local versus systemic host responses was attempted.

\section{Mean relative growth rate (MRGR)}

Nymphs aged 3-4 days were individually weighed on a microbalance (Mettler-Toledo MX5) before each was placed onto the youngest, fully expanded trifoliate leaf of a 3-weekold plant. One aphid was placed per plant to allow individual growth rates to be calculated. Twenty replicates of each plant genotype were used for each aphid clone. The weights of all surviving aphids were measured again 3 days later, and numbers surviving were noted. The MRGR was calculated 
Table 2. Medicago truncatula host plant genotypes tested. (A) Cultivated genotypes; (B) natural accessions.

(A) Cultivar pairs and progenitor genotypes

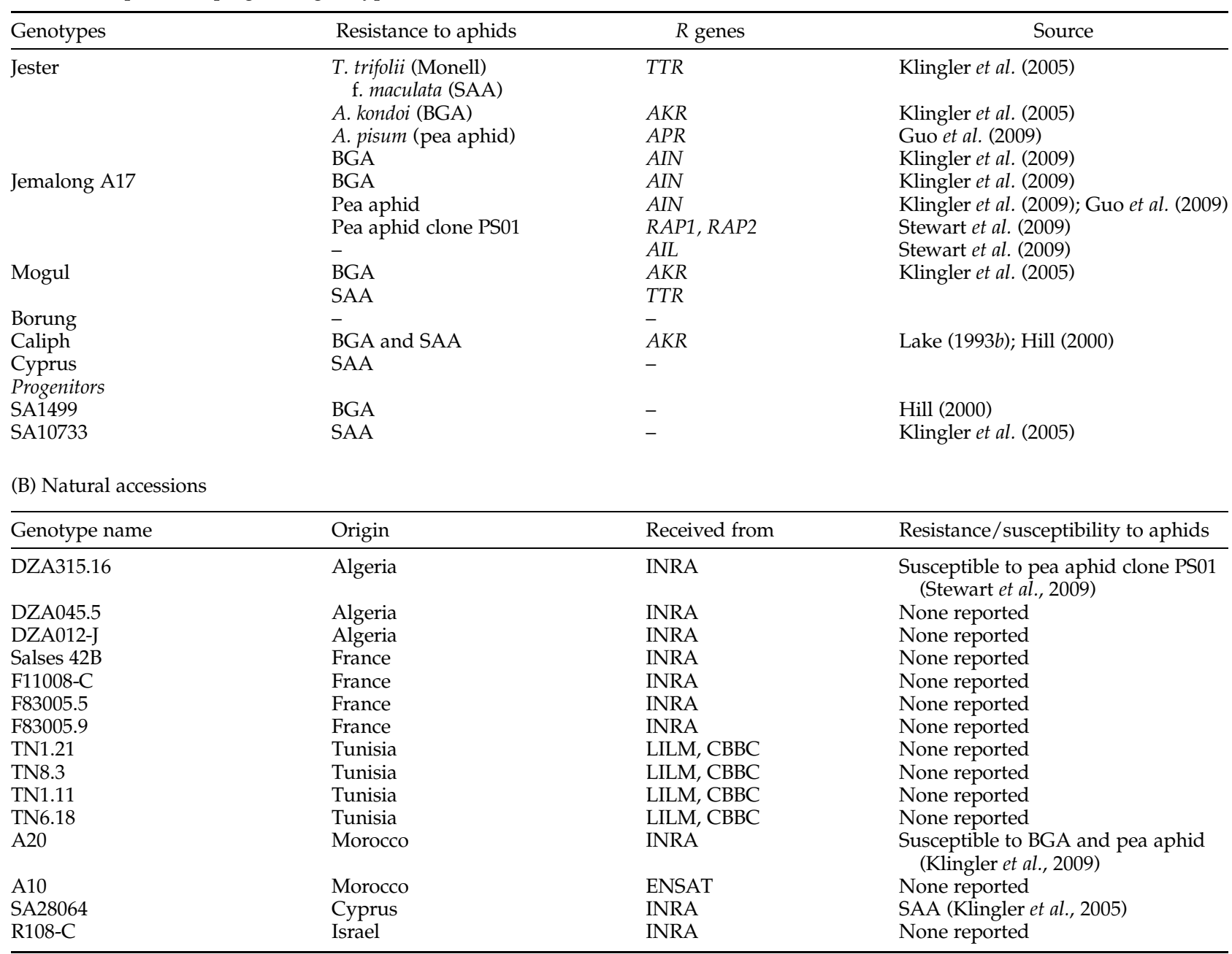

BGA, blue-green aphid; SAA, spotted alfalfa aphid;,$- R$ genes not reported.

INRA: Institut National de la Recherche Agronomique, France; LILM, CBBC: Laboratoire Interactions Legumineuses - Microorganismes, Centre de Biotechnologie, Technopole de Bordj-Cedria, Tunisia; ENSAT: Laboratoire d'Ecologie Fonctionnelle, Ecole Nationale Superieure Agronomique de Toulouse, Tolosan, France.

using the following formula (Radford, 1967; Leather \& Dixon, 1984):

$$
\operatorname{MRGR}=\left(\log W_{\text {final }}-\log W_{\text {original }}\right) /\left(t_{2}-t_{1}\right),
$$

where $W_{\text {original }}$ is the weight at time $t_{1}$ and $W_{\text {final }}$ is the weight at $t_{2}, t_{1}$ is the time of initial measurement; $t_{2}$ is the time of final measurement, in this case 3 days later.

\section{Statistical analysis}

Hierarchical bi-clustering analysis was applied to datasets for survival, reproduction, chlorosis and necrosis, using the Statistixl 1.9 Add-in package within Excel. The aim was to provide visualizations of these complex datasets, and to enable interpretations of data beyond that achievable from univariate ANOVA and multiple pairwise correlation approaches. Groupings of performance and phenotypic data can potentially be used to infer genotypic relationships among aphid or host types. Reproduction data had a significantly skewed distribution and values were square root transformed prior to clustering in order to generate more equalized variances. Chlorosis and necrosis \% values were arcsin square root transformed for clustering. Pearson $R$ pairwise correlation matrices are given for all datasets in Supplementary tables S1S3, except for host genotype correlations for chlorosis and necrosis, where several asymptomatic genotypes precluded calculation of a complete matrix. Distance metrics and clustering routines were selected on basis of the maximal cophenetic correlations between the dendrogram distances and the original unmodelled pairwise data correlations (Supplementary table S4). For survival data, the Euclidean distance metric and the Lance \& Williams flexible clustering method were applied with combinatorial variables 
Table 3. Two-way ANOVA summary for the four measured variables: survival, reproduction, chlorosis and necrosis.

\begin{tabular}{|c|c|c|c|c|c|c|}
\hline Variable & Source & Type III SS & $\mathrm{df}$ & MS & $F$ & $P$ \\
\hline Survival & $\begin{array}{l}\text { Host } \\
\text { Clone } \\
\text { Host } \times \text { clone } \\
\text { Error }\end{array}$ & $\begin{array}{l}2818.6 \\
3324.4 \\
2116.4 \\
1422.3\end{array}$ & $\begin{array}{r}24 \\
7 \\
168 \\
1800\end{array}$ & $\begin{array}{c}117.4 \\
474.9 \\
12.6 \\
0.79\end{array}$ & $\begin{array}{r}148.6 \\
601.0 \\
15.9\end{array}$ & $\begin{array}{l}0 \\
0 \\
3.0 \mathrm{E}-253\end{array}$ \\
\hline Reproduction & $\begin{array}{l}\text { Host } \\
\text { Clone } \\
\text { Host } \times \text { clone } \\
\text { Error }\end{array}$ & $\begin{array}{l}405,936.6 \\
107,872.5 \\
195,508.9 \\
155,574.9\end{array}$ & $\begin{array}{r}24 \\
7 \\
168 \\
1800\end{array}$ & $\begin{array}{r}16914.0 \\
15410.4 \\
1163.7 \\
86.4\end{array}$ & $\begin{array}{r}195.7 \\
178.3 \\
13.5\end{array}$ & $\begin{array}{l}0 \\
1.0 \mathrm{E}-200 \\
1.1 \mathrm{E}-217\end{array}$ \\
\hline Chlorosis & $\begin{array}{l}\text { Host } \\
\text { Clone } \\
\text { Host } \times \text { clone } \\
\text { Error }\end{array}$ & $\begin{array}{r}86.0 \\
102.6 \\
88.4 \\
65.0\end{array}$ & $\begin{array}{r}24 \\
7 \\
168 \\
1800\end{array}$ & $\begin{array}{r}3.58 \\
14.66 \\
0.53 \\
0.04\end{array}$ & $\begin{array}{r}99.3 \\
406.1 \\
14.6\end{array}$ & $\begin{array}{l}5.2 \mathrm{E}-308 \\
0 \\
5.9 \mathrm{E}-234\end{array}$ \\
\hline Necrosis & $\begin{array}{l}\text { Host } \\
\text { Clone } \\
\text { Host } \times \text { clone } \\
\text { Error }\end{array}$ & $\begin{array}{r}272.9 \\
9.4 \\
67.1 \\
63.2\end{array}$ & $\begin{array}{r}24 \\
7 \\
168 \\
1800\end{array}$ & $\begin{array}{r}11.37 \\
1.35 \\
0.40 \\
0.04\end{array}$ & $\begin{array}{r}323.8 \\
38.4 \\
11.4\end{array}$ & $\begin{array}{l}0 \\
1.6 \mathrm{E}-50 \\
3.0 \mathrm{E}-185\end{array}$ \\
\hline
\end{tabular}

$\alpha_{1}=\alpha_{2}=0.625 ; \beta=-0.25 ; \gamma=0$. For all other datasets, the squared Euclidean distance was applied with clustering by Ward's minimum variance method (Everitt, 1980). Relationships between aphid survival and host phenotypic responses were assessed from Pearson's correlation coefficients on arcsin square-root transformed data (Supplementary table S5). Analysis of MRGR was done by two-way ANOVA in Graphpad Prism 5.0 (Supplementary table S6). Significance comparisons were done using Tukey's post-hoc test.

\section{Results}

Species-wide virulence and resistance relationships

Based on the different sites and host species from which the aphid clones were originally collected, and the wide range of host germplasm, we predicted substantial diversity in aphid-host compatibility responses across the 200 combinations tested. In addition to $23 \mathrm{Mt}$ genotypes, we included the close relative $M$. sativa from which several of the aphid clones were collected, and also the universal pea aphid host, $V$. faba. ANOVA indicates that most of the variation in aphid performance is associated with aphid and host genotypes and with genotype $\times$ genotype interactions (table 3 )

Bi-clustering analysis was applied to survival and reproduction datasets (fig. 1; Supplementary figs S1-S6). The clustering dendrograms and associated heatmaps enabled visualization of overall virulence trends across the set of aphid clones, and overall resistance/susceptibility across the host genotypes. Many groupings and patterns were evident, ranging from highly virulent aphids on almost all hosts, to clones that were compatible with only a few host genotypes. Similarly, some Medicago hosts were almost as susceptible as the $V$. faba compatible control, whereas others exhibited strong resistance to the majority of aphid clones. In the survival analysis (fig. 1A), seven host clusters were identified. Clusters 1 and 2 displayed clone-specific resistance to JF01/29 and PS01, respectively, but were susceptible to most other clones. Cluster 3 uniquely showed susceptibility to N127. Cluster 4 was susceptible to most clones, whereas Cluster 5 displayed resistance to all except LL01 and N116. Reproduction data (fig. 1B) grouped into three major host clusters: Cluster 2 showed strong resistance to most clones, whereas Cluster 3 was highly susceptible. Cluster 1 was more diverse, indicative of multiple genotype $\times$ genotype interactions, manifest as clone-specific resistance. Complete resistance was found only on one host, DZA012-J, due to a failure to reproduce across all pea aphid clones.

Clone N127 was the least virulent overall, being moderately successful only on two host lines (R108-C and TN8.3; fig. 1). At the other end of the spectrum, clones LL01 and N116 were clearly the most virulent, with reduced survival $(<60 \%)$ found on only two host genotypes for each clone (fig. 1A, Clusters 6 and 7, respectively), and reduced reproduction on a slightly wider range of hosts mostly within Cluster 2 in fig. 1B. Although LL01 and N116 have strong similarities, they do have contrasting performance on certain Mt genotypes. Likewise, other pairs of pea aphid clones could be differentiated by their performance on one or more Medicago genotype. The unique performance profiles are consistent with molecular genotyping data (J.-C. Simon, personal communication) showing that clones are genetically differentiated and can be classified into different species-level biotypes (table 1).

The three pairs of Australian cultivars that represent genotypes with contrasting resistance-susceptibility to a range of aphid species did not show corresponding resistance to European pea aphid clones. Indeed, Caliph and Cyprus performed nearly identically, being susceptible to all clones except N127 (fig. 1A, Cluster 4; fig. 1B, Cluster 3). Mogul and Borung displayed the clone-specific resistance patterns that grouped closely together (fig. 1A, Cluster 5; fig. 1B, Cluster 1), with both showing significant resistance to all clones except N116 and LL01. The cultivar Jester is derived substantially from A17 and displays increased resistance to Australian pea aphid biotypes (Gao et al., 2008; Guo et al., 2009). However, Jester was not more resistant than A17 when challenged with the European pea aphid clones, and in fact had significantly greater susceptibility than A17 to several clones (Supplementary fig. S1), and thus featured in different clusters in both plots (fig. 1).

In addition to the performance data represented by survival and reproduction, we examined aphid growth rates over the first 3 days of infestation in subsets of the pea aphid-Mt combinations. Growth rate can be a more sensitive measure than survival, especially where there is sublethal incompatibility. The first experiment compared all clones 

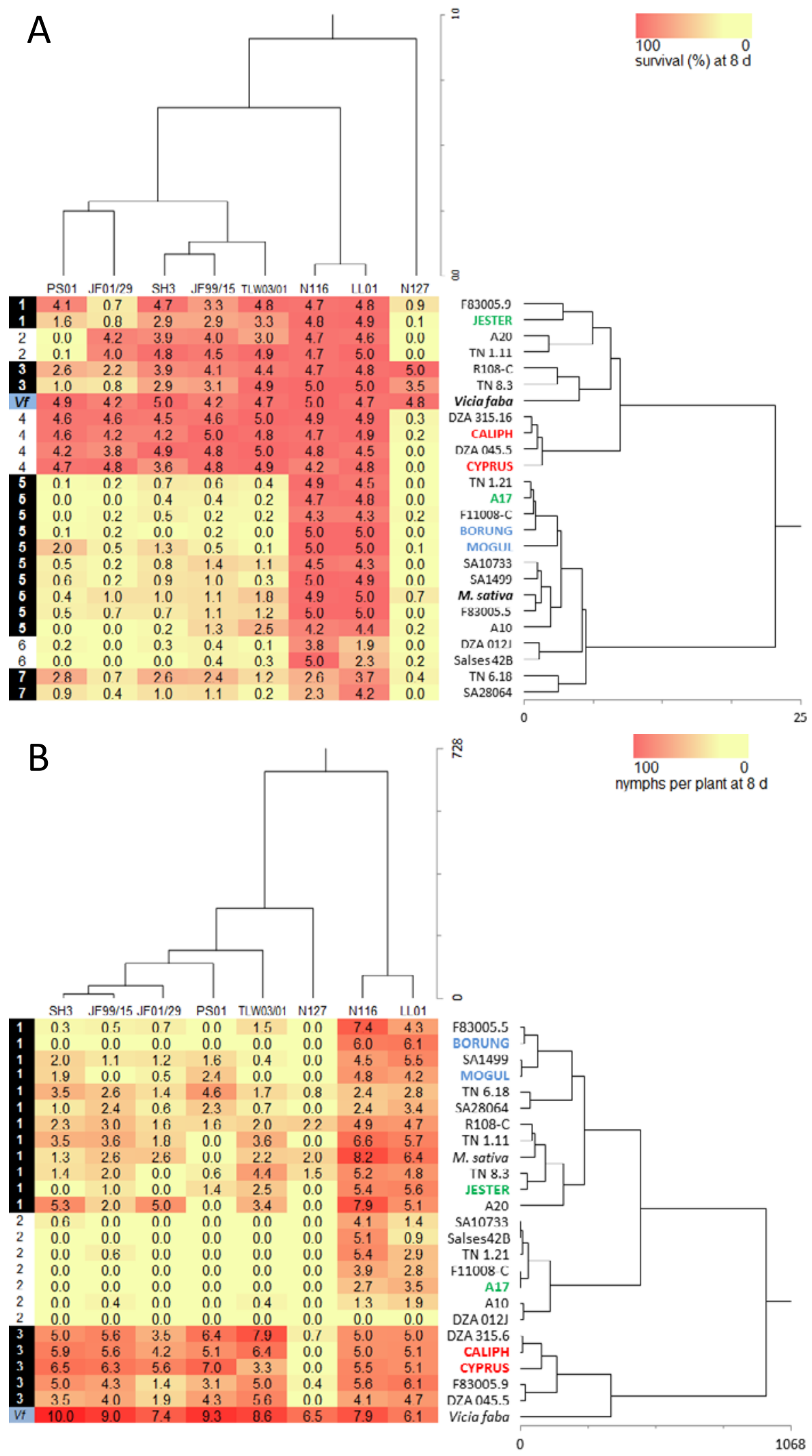

Fig. 1. Survival and clonal reproduction of pea aphid clones on a range of Medicago genotypes. Data presented as bi-clustering analyses with aphid clones as columns and host genotypes as rows. (A) Values are mean adult survival at 8 days after infestation with five nymphs 

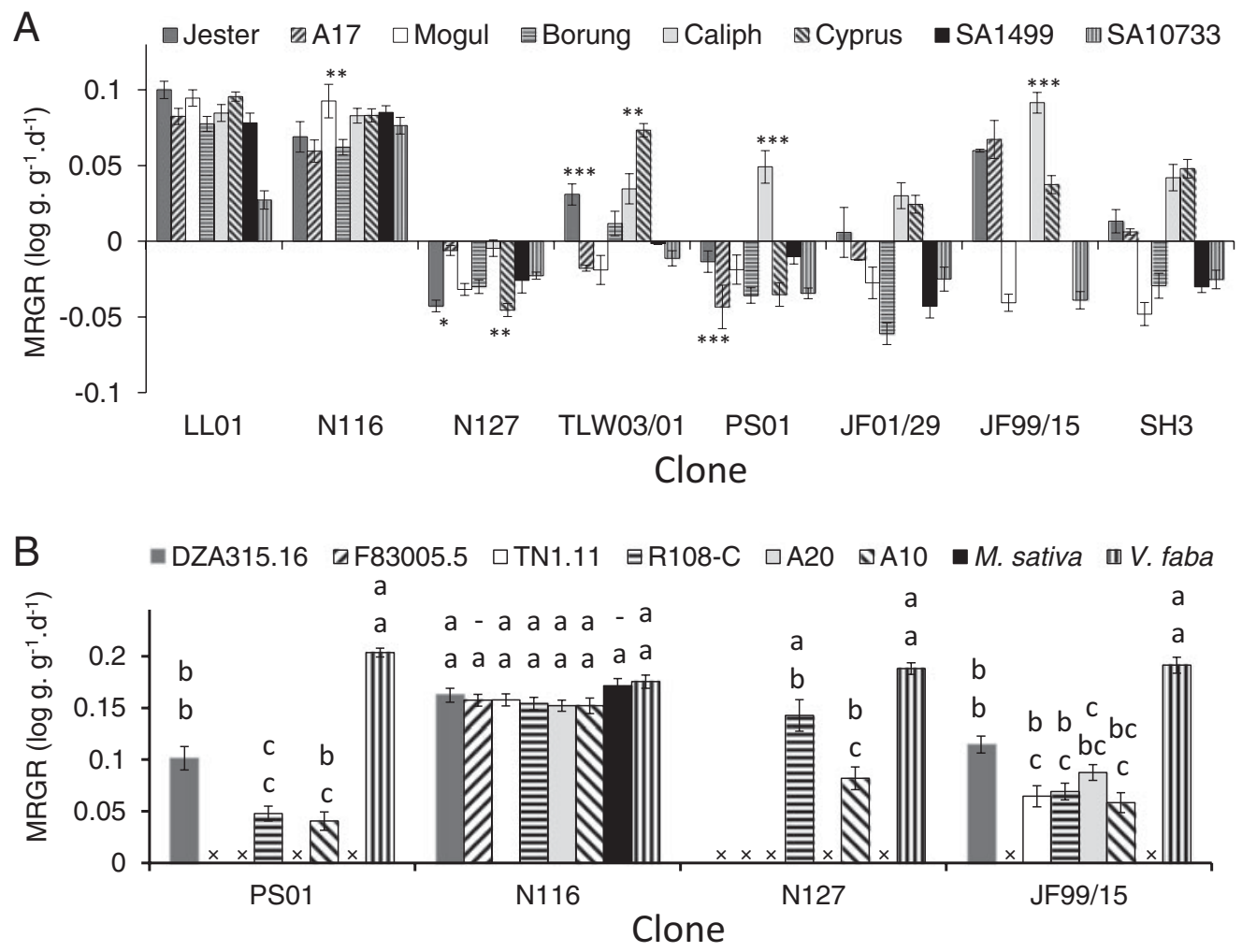

Fig. 2. Mean Relative Growth Rate (MRGR) of surviving adult aphids on selected host genotypes. (A) Three resistant-susceptible pairs of closely related Mt cultivars: Jester-A17, Mogul-Borung and Caliph-Cyprus, and two of their progenitor lines, SA1499 and SA10733; ${ }^{*} P<0.05$; ${ }^{* *} P<0.01 ;{ }^{* * *} P<0.001$ from Tukey's post-hoc test for within-clone comparisons of host pairs. (B) Subset of natural accessions of Mt. Values recorded 3 days after infestation. Bars show mean \pm SE (1 nymph per plant, 20 replications). Positive and negative values represent weight gain and loss, respectively. Missing bars marked ' $x$ ' represent $<50 \%$ aphid survival, where MRGR was deemed unreliable. Different letters above bars indicate significant difference at $P<0.05$ from Tukey's post-hoc test, for within-clone, between-host contrasts (upper letter) and within-host, between-clone contrasts (lower letter).

on the three pairs of related resistant-susceptible cultivars, and also included two of their progenitor lines (fig. 2A). Most aphid-host combinations $(94 \%)$ had sufficient survival at 3 days to enable accurate MRGR estimations. Consistent with the survival and reproduction data, the highly virulent clones LL01 and N116 showed high positive MRGR on almost all hosts, whereas the poorly adapted clone N127 had negative MRGR in every case. The other five clones displayed hostspecific MRGRs, ranging from high positive to strongly negative. However, there was little evidence overall for lower MRGR values on any of the resistant genotypes compared with their susceptible relatives. The second experiment examined responses of four pea aphid clones on six natural Mt accessions, together with $M$. sativa and $V$. faba (fig. 2B). The combinations were selected to represent strongly and weakly virulent clones, together with strongly and weakly resistant host genotypes. Here, survival rates were much more varied at 3 days, with over a third of combinations being excluded from analysis because of low survival. This variability may reflect the greater genetic diversity of the natural versus cultivated accessions. Where MRGR was recorded reliably, most of the values again correlated well with previous survival data. The two exceptions were N127 and JF99/15, both on A10 host, which had positive MRGR yet were previously scored as incompatible.

\section{Host responses}

In parallel with monitoring aphid performance, two indicators of host responses to infestation were quantified: aphid-induced chlorosis and aphid-induced necrosis. A wide range of symptom frequencies was recorded, often showing consistent within-genotype responses for host and/or aphid (fig. 3). Thus, some host genotypes displayed chlorosis or necrosis to all aphids, and some displayed none. Equally, some aphid biotypes induced symptoms on all or nearly all

per plant; heatmap scaling is from $0 \%$ survival (pale yellow) to $100 \%$ (red); (B) number of new nymphs produced after 8 days, shown as square-root values, used in clustering procedure. Actual mean numbers per plant ranged from 0 (pale yellow) to 100 (red). Host genotypes sharing same numbers in first column represent clusters with similar resistance/susceptibility profiles. Vicia faba was included as a universally susceptible control. Mt genotypes highlighted capitalized in different colours represent resistant-susceptible pairs of lines reported in response to Australian pea aphid biotypes. $N=10$ plants per aphid clone, with five aphids per plant. 


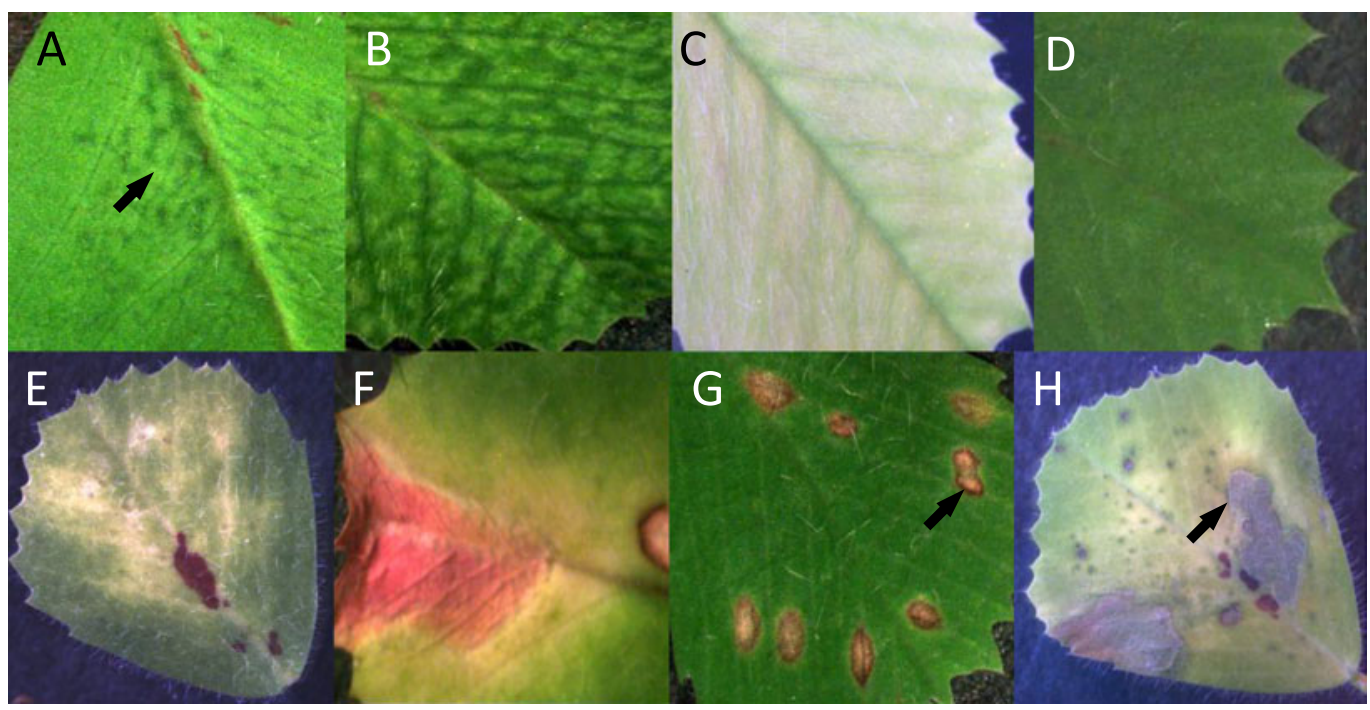

Fig. 3. Medicago truncatula host phenotypic symptoms following pea aphid infestation. (A-C) Stages of interveinal chlorosis induced by clone N116, from (A) early interveinal chlorotic response (arrowed) to (B) interveinal chlorosis spread to entire leaf and (C) entire leaf yellow; (D) healthy leaf; (E) chlorosis induced by all other clones; (F) senescent leaf that will eventually abscise. (G, H) Necrosis induced by aphid feeding, ranging from $(\mathrm{G})$ leaves with small lesions (arrowed) to $(\mathrm{H})$ larger necrotic patches (arrowed).

hosts, and some induced few or no symptoms. As with aphid performance, ANOVA indicates that most of the variation in host responses is genetically based (table 3). Bi-clustering analysis was again applied to enable visualization of overall groupings and patterns, and resolved chlorosis and necrosis responses into six and four distinct clusters, respectively (fig. 4).

\section{Chlorosis}

Of the 25 host genotypes tested, only two (R108-C and F83005.9; fig. 4A, Cluster 6) displayed extensive chlorosis in response to all eight aphid clones), whereas minimal or no chlorosis was found on three Mt genotypes (A10, A20 and TN6.18; Cluster 4) and on $V$. faba. Wide variation across the aphid clones was also found, with three (JF01/29, N127 and PS01) inducing few or no symptoms on any host apart from the universally chlorotic R108-C and F83005.9. At the other extreme, N116-induced chlorosis on all Medicago lines except the minimally responsive Cluster 4 mentioned above. The chlorotic phenotype resulting from N116 was noticeably distinct from the generalized yellowing of the leaf around aphid feeding sites induced by other clones (fig. 3E), instead displaying marked interveinal chlorosis or mosaic symptoms (fig. 3A, B). If the atypical N116-induced chlorosis is excluded, then several further groupings can be defined: 14 hosts (Clusters $1,2,4)$ had low chlorosis $(\leq 20 \%)$, whereas a biotype-specific highly chlorotic cluster (\#3) was found for TLW03/01 (four host genotypes that did not respond to the other biotypes) and another cluster (\#5) centred on very strong symptoms from LL01, containing four genotypes that also responded but less strongly to other biotypes (fig. 4A). Some differences were found between the symptoms on the closely related pairs of host genotypes, with certain clones inducing significantly more chlorosis on Jester than on A17 $(P<0.05)$, and likewise a higher incidence on Cyprus than on Caliph $(P<0.001)$ (fig. 4A, Supplementary table S5A).
Chlorosis was positively correlated with aphid survival when assessed across all aphid-host combinations $(r=0.56$, $P<0.001)$. However, this relationship was attributable to eight Mt host lines and $M$. sativa $\left(R^{2}=0.75-0.99 ; P<0.05\right.$ to $P<0.001)$ with no significant correlation for the majority of host genotypes (Supplementary table S5B). The groupings found for chlorosis (fig. 4A) had no obvious correspondence to those found for aphid survival and reproduction (fig. 1), either in clusters of aphid biotypes or in clusters of host genotypes.

\section{Necrosis}

Based on cluster analysis (fig. 4B), the great diversity in incidence of aphid-induced necrosis appeared to be largely determined by the host genotype. Symptoms varied from multiple small lesions (fig. 3G) at or near presumed probing or feeding sites to broad necrotic patches on infested leaves (fig. 3H). Five genotypes (fig. 4B, Cluster 2) displayed extensive lesions in response to all or nearly all aphid clones, whereas nearly half ( 12 out of 25 ; Cluster 3 ) of the tested hosts showed minimal or no necrosis, as did M. sativa and $V$. faba. The remaining eight host genotypes (Clusters 1,4) showed symptoms that were dependent on the aphid-host combination, and ranged from completely absent to $100 \%$. With the exception of $V$. faba, only one host genotype (A20) showed neither chlorosis nor necrosis in response to any aphid clone, whereas all clones induced both chlorosis and necrosis on F83005.9.

Each of the three closely related host genotype pairs showed very similar necrosis responses: Caliph and Cyprus had virtually no symptoms, Mogul and Borung had strong necrosis induced by all clones, whereas Jester and A17 showed nearly identical clone-specific responses.

Medicago genotypes with necrosis were observed in all categories ranging from highly resistant to highly susceptible. Aphid-induced necrosis was essentially unrelated to aphid survival across all combinations tested (Pearson $r=-0.034$, 

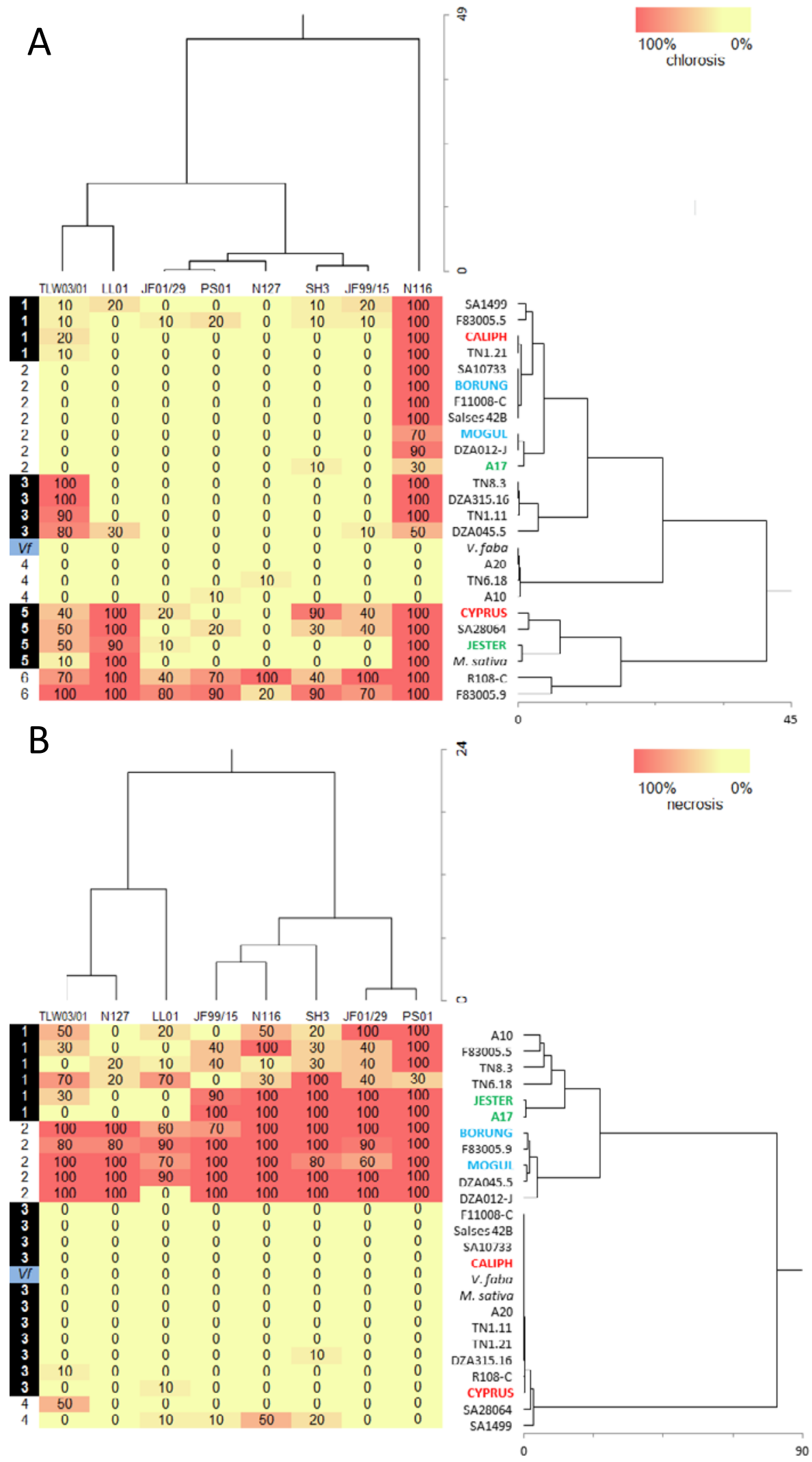

Fig. 4. Host responses to pea aphid infestation. Data presented as bi-clustering analyses with aphid clones as columns and host genotypes as rows. (A) chlorosis; (B) necrosis. Both measured as \% of plants showing symptoms 8 days after infestation. Other details as for fig. 1. Host genotypes sharing same numbers in first column represent clusters with similar host response profiles. 
$P>0.05$; Supplementary table S5). Overall the strong groupings of host genotypes for necrosis symptoms (fig. 4) did not match the clusters delineated for survival and reproduction (fig. 1), although significant relationships were detected for a few individual combinations (Supplementary table S5).

\section{Discussion}

\section{Extensive virulence and resistance diversity in both pea aphid and Medicago}

This work has uncovered wide variation in performance of eight different pea aphid clones across a set of $23 \mathrm{Mt}$ host genotypes and on $M$. sativa. Aphid virulence and host resistance were highly dependent on genotype of both aphid and host, with great diversity in host-specific pea aphid performance and clone-specific resistance in Mt. Each clonal population differed in apparent adaptation across the range of tested host genotypes, strongly indicating that they are all genetically distinct, and suggesting substantial genetic complexity among both aphid clones and hosts. Most clones harboured secondary endosymbionts, but presence or absence of these species showed no obvious correlation with host compatibility. The one clone that lacked secondary endosymbionts, LL01, was highly virulent on most hosts, similar to N116 which carried two detected secondary endosymbiont species. Future studies could evaluate cured and re-infected clones for effects on performance.

Extensive genetic diversity in species-level host adaptation has previously been reported in pea aphid (Via, 1999; Frantz et al., 2006. Peccoud et al., 2009), but less is known about the impact of intra-specific host variation. The many compatible interactions indicate that even in generally resistant Mt lines, the resistance rarely extends to all clones. None of the clones tested were known to have had previous exposure to $\mathrm{Mt}$, which is not grown in the UK or Ireland, and the sole French clone tested here was from outside the natural distribution range of Mt. Potential for virulence on Mt thus appears widespread among pea aphid populations even without recent prior adaptation to this host species, and geographic migration might readily introduce highly compatible biotypes onto Mt. The range of performance of these pea aphid clones on $\mathrm{Mt}$ raises questions as to how such extensive variation is generated and sustained in the absence of Mt-specific selection pressure. Currently there is no published mapping or marker data to indicate the degree of genetic similarity between Australian and European pea aphid. However, there may have been no recent recombination between these geographically isolated populations, and therefore divergent host adaptation might be predicted.

In the field, approximately $10 \%$ inter-host migration has been estimated (Via, 1999). Genetically distinct sympatric populations of aphids can thus interbreed, resulting in recombinant hybrids with differing levels of host adaptation. In some cases, fitness of such recombinants on the original hosts is lower than for the specialized parent (Via et al., 2000). The universal virulence of the pea aphid clones on $V$. faba, regardless of their original host, suggests that this species could be a common host for pea aphid populations, potentially restricting further divergence towards speciation (Ferrari et al., 2008).

There was no simple relationship here between original host plant from which a clone was collected and subsequent performance under experimental conditions. However, some patterns emerged that enable deductions about the underlying genetic basis.

1. Two aphid clones JF99/15 and SH3 had very highly correlated although not identical virulence profiles across the panel of host genotypes, yet were originally collected from different host species, Trifolium pratense and Pisum sativum, respectively and were classified into different host race groupings.

2. Clone TLW03/01 performed much better on several Mt genotypes than on its original host, $M$. sativa. This clone could have been a migrant from a different host species, consistent with its molecular genotype being indicative of a Trifolium host race and thus it performed poorly on $M$. sativa, while showing high fitness on other hosts (Ferrari et al., 2008). Additionally, the $M$. sativa genotype used in the present work may differ in resistance genes from the original source plant from which TLW03/01 was collected.

3. Clone N127, also from M. sativa, was weakly virulent on this species and failed to thrive on all except two Mt genotypes (TN8.3 and R108-C). The basis of this highly restricted host adaptation is unclear, but at least for R108-C, may relate more to this host being among the most susceptible to all tested clones rather than N127 having a particular virulence factor. N127 was classified as a Medicago lupulina biotype, and thus may also have been a migrant on $M$. sativa.

4. At the opposite extreme, two clones (N116 and LL01) also collected from $M$. sativa, were both classified by molecular genotyping as $M$. sativa host races, displayed high virulence on this species in our experiments, and performed well on nearly all Mt genotypes. However, N116 and LL01 could be distinguished by differential performance on a small number of hosts, and differed greatly in induction of host phenotype symptoms. On this basis and from molecular marker profiles, the clones appear to be genetically distinct although their evolutionary histories are unknown.

\section{Mechanism of resistance}

Incompatible interactions between pea aphid and $\mathrm{Mt}$ typically result in weight loss leading to early death of avirulent aphids and/or reduced reproduction. Conventionally, antixenosis is defined as the failure to accept and feed on a particular host due to perception of negative cues or absence of positive cues (Smith \& Clement, 2012). It is possible that antixenosis mechanisms were operating in aphids on resistant Mt lines leading to plant rejection prior to feeding and death due to starvation, akin to a previous report by Caillaud and Via (2000). Alternatively, low survival and/or reproduction could be attributed to antibiotic influences ingested from the host during feeding. A further possible type of antibiosis was seen on DZA012-J where some clones survived but, uniquely, none reproduced. Choice experiments would allow assessment of differential host acceptance, and would enable clearer deductions about the nature of the incompatibilities detected. In this context, it has recently been suggested that revision of the categories of resistance and tolerance is required in light of emergence of better understanding of underlying molecular mechanisms (Stout, 2013). Future explorations could assess host variation in positive factors such as nutrients and phagostimulants (Auclair, 1963), alongside sensory differences among aphid populations. 


\section{Sources of resistance}

In this study, overall resistance was strongest in genotype DZA012-J. Although moderate survival of LL01 and N116 was noted, DZA012-J was the only host on which no aphid reproduction was found, and may therefore represent a useful source of germplasm for broadly based pea aphid resistance breeding. However, it cannot be predicted whether such resistance would be effective against all global pea aphid biotypes, nor whether it would rapidly break down due to aphid evolution through recombination and mutation. Several other Mt genotypes were strongly resistant to several clones, but N116 and LL01 both showed some survival and reproduction on these lines. An informative future experiment would be assessment of whether resistance of these genotypes is also effective against pea aphid clones collected directly from Mt.

The performance of the three pea aphid resistantsusceptible pairs of Australian Mt cultivars did not conform to expectations. Overall performance was not significantly different within two of the pairs (Mogul-Borung; CaliphCyprus). We found that, surprisingly, the enhanced resistance reported against Australian pea aphid clones in Jester is not effective against the European clones, and resistance to three of the pea aphid clones was in fact moderately reduced in Jester compared with its A17 parent. As Jester has a genetic make-up that comprises 89\% from A17 (Hill, 2000), it appears that some A17-derived pea aphid resistance has been lost during breeding and selection of Jester. Nonetheless, Jester displays resistance to SAA and BGA, in addition to Australian pea aphid (Gao et al., 2008).

Given that the resistant-susceptible Mt pairs each differ in one of more of the genes TTR, $A K R$ and $A P R$, it is likely that additional genes in these lines are controlling resistance to European pea aphid clones. Race-specific variation in compatibility with different genotypes within a host species has been reported for other insect pests such as greenbug (Kerns et al., 1989), and resistance genes in wheat can vary in effectiveness against different Russian wheat aphid clones (Burd et al., 2006). Two independent loci, RAP1 and RAP2, in A17 confer resistance to specific pea aphid clones including PS01 (Stewart et al., 2009) and JF01/29 (Stewart, 2010) but it has yet to be established whether these genes are also responsible for A17 resistance to other clones tested here. For the natural accessions of $\mathrm{Mt}$, clustering of host genotypes did not correlate with geographic sites of origin. For example Algerian, Tunisian and French genotypes were represented across highly susceptible, moderately resistant and highly resistant clusters.

\section{Host responses}

Possible relationships between host symptoms and aphid performance were evaluated. The lack of overall significant patterns indicates that the presence or absence of chlorosis or necrosis was not universally necessary for determining compatible or incompatible interactions. Moreover, incidence of chlorosis and necrosis was not correlated, and therefore appear to be two independent forms of host response to aphids. Aphid survival was positively correlated to chlorosis in six Medicago lines, but for all other host genotypes there was no significant relationship.

Although previous work has frequently indicated an association of HR-like necrotic symptoms with incompatible aphid interactions (Belefant-Miller et al., 1994; Moloi \& van der Westhuizen, 2006; Klingler et al., 2009; Villada et al., 2009), we found essentially no significant relationship between aphid survival and necrosis across the Mt germplasm tested here (Supplementary table S1), consistent with some other previous reports (Martinez de Ilarduya et al., 2003; Stewart et al., 2009). For example, clones N116 and LL01 were highly virulent on most hosts, yet N116 induced strong necrosis on certain compatible lines (A17, Jester, F83005.5), where LL01 caused no necrosis. No necrosis was observed on several Mt genotypes (fig. 4B, Cluster 3) nor on $M$. sativa in response to any of the aphid clones, yet each of these hosts displayed resistance to more than one clone. Clearly, aphid-induced necrosis is neither necessary for, nor a good predictor of, genotype-specific resistance. Although the final necrotic cell death symptoms may not be essential for resistance, it remains possible that some upstream processes are shared, such as metabolic changes and signalling that lead to the expression of defence genes.

\section{Conclusions}

This exploration of pea aphid performance on Medicago has led to a number of conclusions:

1. Pea aphid shows highly significant inter-clonal variation in performance on unfamiliar Mt host genotypes, with each clone likely representing a unique virulence genotype or biotype.

2. All clones survived at least partially on two or more Mt genotypes, with $M$. sativa biotypes LL01 and N116 being the most virulent, and N127 appearing poorly adapted to both Mt and M. sativa.

3. Host resistance varied greatly, with only one genotype displaying complete resistance to all tested clones.

4. Host chlorosis and necrosis were generally poorly correlated with aphid performance.

Strategies for enhancement of aphid resistance in crop and pasture species include incorporation of additional $R$ genes available in the germplasm pool. Although only a few $R$ gene loci have been defined in $\mathrm{Mt}$, the present study has indicated great diversity in adaptation among different pea aphid biotypes and in resistance across the panel of host genotypes. We thus advocate that crop improvement programmes screening for aphid resistance should ideally include multiple independent clones for each aphid species. Given the highly virulent nature of clones such as N116 and LL01, and the likely polygenic nature of aphid-host interactions, robust and durable resistance may require $R$ gene stacking.

The pea aphid-Mt system is presently the only aphid-host model, where both genome sequences have been published (The International Aphid Genomics Consortium, 2010; Young et al., 2011). Inbred derivatives of 14 of the Mt lines tested here are represented in the deep resequencing HapMap set (medicagohapmap.org), and most of the other lines have been similarly resequenced at lower density (Branca et al., 2011). In addition, several sets of recombinant inbred lines (RILs) are available, including some developed from genotypes tested here (montpellier.inra.fr/BRC-MTR/accueil. php), that represent powerful tools for isolating the molecular determinants of resistance variation. Resequencing of multiple pea aphid clones has also recently been reported (Bickel et al., 2013; Gouin et al., 2013). Despite the availability of such 
resources, little is yet known about aphid effectors and other genetic factors that determine compatibility. Although controlled genetic experiments with aphids are time-consuming and labour intensive, they may be highly rewarding for uncovering the heritable basis of virulence and avirulence, and allowing estimation of rates of recombination.

\section{Supplementary Material}

The supplementary material for this article can be found at http://www.journals.cambridge.org/BER

\section{Acknowledgements}

We thank Sophie Stewart, Simon Hodge and Martin Selby for valuable assistance, and Tilly Collins for statistical advice. Jean-Christophe Simon is gratefully acknowledged for genotyping the aphid clones, as are Alison Karley and Angela Douglas for aphid endosymbiont data. SK was supported by a Beit Scientific Research Fellowship at Imperial College London.

\section{References}

Auclair, J.L. (1963) Aphid feeding and nutrition. Annual Review of Entomology 8, 439-490.

Belefant-Miller, H., Porter, D.R., Pierce, M.L. \& Mort, A.J. (1994) An early indicator of resistance in barley to Russian wheat aphid. Plant Physiology 105, 1289-1294.

Berzonsky, W.A., HongJian, D., Haley, S.D., Harris, M.O. \& Lamb, R.J. (2003) Breeding wheat for resistance to insects. Plant Breeding Reviews 22, 221-296.

Bickel, R.D., Dunham, J.P., \& Brisson, J.A. (2013) Widespread selection across coding and noncoding DNA in the pea aphid genome. Genes Genomes Genetics 3, 993-1001.

Blackman, R.L. \& Eastop, V.F. (2000) Aphids on the World's Crops. an Identification and Information Guide. Chichester, UK, Wiley.

Boyko, E.V., Smith, C.M., Thara, V.K., Bruno, J.M., Deng, Y.P., Starkey, S.R. \& Klaahsen, D.L. (2006) Molecular basis of plant gene expression during aphid invasion; wheat Pto- and Pti-like sequences are involved in interactions between wheat and Russian wheat aphid (Homoptera; Aphididae). Journal of Economical Entomology 99, 1430-1445.

Branca, A., Paape, T.D., Zhou, P., Briskine, R., Farmer, A.D., Mudge, J., Bharti, A.K., Woodward, J.E., May, G.D., Gentzbittel, L., Ben, C., Denny, R., Sadowsky, M.J., Ronfort, J., Bataillon, T., Young, N.D. \& Tiffin, P. (2011) Whole-genome nucleotide diversity, recombination, and linkage disequilibrium in the model legume Medicago truncatula. Proceedings of the National Academy of Sciences of the United States of America 108, E864-E870.

Burd, J., Porter, D., Puterka, G., Haley, S. \& Peairs, F. (2006) Biotypic variation among north American Russian wheat aphid (Homoptera: Aphididae) populations. Journal of Economic Entomology 99, 1862-1869.

Caillaud, M.C. \& Via, S. (2000) Specialized feeding behavior influences both ecological specialization and assortative mating in sympatric host races of pea aphids. The American Naturalist 156, 606-621.

Chen, M-S. (2008) Inducible direct plant defense against insect herbivores: a review. Insect Science 15, 101-114.

Crawford, E., Lake, A. \& Boyce, K. (1989) Breeding annual Medicago species for semiarid conditions in Southern Australia. Plant Molecular Biology 60, 519-531.
Everitt, B. (1980) Cluster Analysis. New York, USA, Halsted Press. Ferrari, J., Godfray, H.C.J., Faulconbridge, A.S., Prior, K. \& Via, S. (2006) Population differentiation and genetic variation in host choice among pea aphids from eight host plant genera. Evolution 60, 1574-1584.

Ferrari, J., Via, S. \& Godfray, H.C.J. (2008) Population differentiation and genetic variation in performance on eight hosts in the pea aphid complex. Evolution 62, 2508-2524.

Frantz, A., Plantegenest, M., Mieuzet, L. \& Simon, J.C. (2006) Ecological specialization correlates with genotypic differentiation in sympatric host-populations of the pea aphid. Journal of Evolutionary Biology 19, 392-401.

Gao, L.L., Horbury, R., Nair, R.M., Singh, K.B. \& Edwards, O.R. (2007) Characterization of multiple aphid species (Hemiptera: Aphididae) in Medicago truncatula. Bulletin of Entomological Research 97, 41-44.

Gao, L.L., Klinger, J.P., Anderson, J.P., Edwards, O.R. \& Singh, K.B. (2008) Characterization of pea aphid resistance in Medicago truncatula. Plant Physiology 146, 996-1009.

Garcia-Brugger, A., Lamotte, O., Vandelle, C., Bourque, S., Lecourieux, D., Poinst, B., Wendehenne, D., \& Pugin, A. (2006) Early signalling events induced by elicitors of plant defences. Molecular Plant Microbe Interactions 19, 711-724.

Gouin, A., Legeai, F., Nouhaud, P., Rizk, G., Simon, J-C. \& Lemaitre, C. (2013) Whole genome re-sequencing: lessons from unmapped reads. In: Journées Ouvertes Biologie Informatique Mathématiques, Toulouse, 1-4 July 2013. http:// hal.inria.fr/hal-00907446.

Guay, J.F., Boudreault, S., Michaud, D. \& Cloutier, C. (2009) Impact of environmental stress on aphid clonal resistance to parasitoids: role of Hamiltonella defensa bacterial symbiosis in association with a new facultative symbiont of the pea aphid. Journal of Insect Science 55, 919-26.

Guo, S., Kamphius, L.G., Gao, L., Edwards, O.R. \& Singh, K.B. (2009) Two independent resistance genes in the Medicago truncatula cultivar Jester confer resistance to two different aphid species of the genus Acrythosiphon. Plant Signalling and Behaviour 4, 1-4.

Guo, S., Kamphius, L.G., Gao, L., Klingler, J.P., Lichtenzveig, J., Edwards, O. \& Singh, K.B. (2012) Identification of distinct quantitative trait loci associated with defence against the closely related aphids Acyrthosiphon pisum and A. kondoi in Medicago truncatula. Journal of Experimental Botany 63, 3913.

Hill, J.R. (2000) Jester. Plant Varieties Journal 13, 40.

The International Aphid Genomics Consortium (2010) Genome sequence of the pea aphid Acyrthosiphon pisum. PLoS Biology 8, e1000313.

Kaloshian, I. (2004) Gene-for-gene resistance: bridging insect pest and pathogen defence. Journal of Chemical Ecology 30, 2421-2439.

Kerns, D.L., Puterka, G.J. \& Peters, D.C. (1989) Intrinsic rate of increase for greenbug (Homoptera: Aphididae) biotypes $\mathrm{E}, \mathrm{F}, \mathrm{G}$, and $\mathrm{H}$ on small grain and sorghum varieties. Environmental Entomology 18, 1074-1077.

Kim, K-S., Hill, C., Hartman, G. \& Diers, B. (2008) Discovery of soybean aphid biotypes. Crop Science 48, 923-928.

Klingler, J.P., Powell, G., Thompson, G.A. \& Isaacs, R. (1998) Phloem specific aphid resistance in Cucumis melo line AR 5: effects on feeding behaviour and performance of Aphis gossypii. Entomologia Experimentalis et Applicata 86, 79-88.

Klingler, J.P., Creasy, R., Gao, L., Nair, R.M., Calix, A.S., Jacob, H.S., Edwards, O.R. \& Singh, K.B. (2005) Aphid resistance in Medicago truncatula involves antixenosis and 
phloem-specific, inducible antibiosis, and maps to a single locus flanked by NBS-LRR resistance gene analogs. Plant Physiology 137, 1445-1455.

Klingler, J.P., Edwards, O.R. \& Singh, K.B. (2007) Independent action and contrasting phenotypes of resistance genes against spotted alfalfa aphid and bluegreen aphid in Medicago truncatula. New Phytologist 173, 630-640.

Klingler, J.P., Nair, R.M., Edwards, O.R. \& Singh, K.B. (2009) A single gene, AIN, in Medicago truncatula mediates a hypersensitive response to both bluegreen aphid and pea aphid, but confers resistance only to bluegreen aphid. Journal of Experimental Botany 60, 4115-4127.

Lake, A. (1993a) Medicago truncatula Gaertn. (barrel medic) cv. Caliph. Australian Journal of Experimental Agriculture 33, 821-822.

Lake, A. (1993b) Medicago truncatula Gaertn. (barrel medic) cv. Mogul. Australian Journal of Experimental Agriculture 33, 823-824.

Leather, S.R. \& Dixon, A.F.G. (1984) Growth and reproductive rates. Entomologia Experimentalis et Applicata 35, 137-140.

Martinez de Ilarduya, O., Xie, Q. \& Kaloshian, I. (2003) Aphidinduced defense responses in Mi-1-mediated compatible and incompatible tomato interactions. Molecular Plant Microbe Interactions 16, 699-708.

Moloi, M.J. \& van der Westhuizen, A.J. (2006) The reactive oxygen species are involved in resistance responses of wheat to the Russian wheat aphid. Plant Physiology 163, 1118-1125.

Müller, F.P. (1962) Biotypen und Unterarten der 'Erbsenlaus' Acyrthosiphon pisum Harris. Zeitschrift für Pflanzenkrankheiten 69, 129-136.

Nombela, G., Williamson, V.M. \& Muñiz, M. (2003) The rootknot nematode resistance gene Mi-1.2 of tomato is responsible for resistance against the whitefly Bemisia tabaci. Molecular Plant Microbe Interactions 16, 645-649.

Peccoud, J., Ollivier, A., Plantegenest, M., \& Simon, J-C. (2009) A continuum of genetic divergence from sympatric host races to species in the pea aphid complex. Proceedings of the National Academy of Sciences of the United States of America 106, 7495-7500.

Puterka, G.J. \& Peters, D.C. (1989) Inheritance of green bug, Schizaphis graminum (Rondani), virulence of $G b 2$ and Gb3 resistance genes in wheat. Genome 32, 109-114.

Puterka, G.J. \& Peters, D.C. (1990) Sexual reproduction and inheritance of virulence in green bug Schizaphis graminum (Rondani). pp. 289-318 in Campbell, R.K. \& Eikenbary, R.D. (Eds) Aphid-Plant Genotype Interaction. New York: Elsevier Press.

Radford, P.J. (1967) Growth analysis formulas - their use and abuse. Crop Science 7, 171-175.

Schoonhoven, L.M., Jemy, T. \& van Loon, J.J.A. (1998) Plants as insect food; not the ideal. pp. 83-120 in Schoonhoven, L.M., van Loon, J.J.A. \& Dicke, M. (Eds) Insect-Plant Biology - From Physiology to Evolution. London: Chapman \& Hall.

Smith, C.M. (2005) Plant Resistance to Arthropods. Dordrecht: Springer.

Smith, C.M., Belay, T., Stauffer, C., Stary, P., Kubeckova, I. \& Starkey, S. (2004) Identification of Russian wheat aphid (Homoptera: Aphididae) populations virulent to the
Dn4 resistance gene. Journal of Economic Entomology 97, 1112-1117.

Smith, C.M. \& Clement, S.L. (2012) Molecular bases of plant resistance to arthropods. Annual Review of Entomology 57, 309-28.

Stewart, S.A. (2010) Exploring effective, clone-specific resistance against the pea aphid (Acyrthosiphon pisum) in Medicago truncatula. PhD thesis, Imperial College London, UK.

Stewart, S.A., Hodge, S., Ismail, N., Mansfield, J.W., Feys, B.J., Prospéri, J.M., Huguet, T., Ben, C., Gentzbittel, L. \& Powell, G. (2009) The RAP1 gene confers effective, racespecific resistance to the pea aphid in Medicago truncatula independent of the hypersensitive reaction. Molecular Plant Microbe Interactions 22, 1645-1655.

Stout, M.J. (2013) Reevaluating the conceptual framework for applied research on host-plant resistance. Insect Science 20, 263-272.

Via, S. (1991) Specialized host plant performance of pea aphid clones is not altered by experience. Ecology 72, 1420-1427.

Via, S. (1999) Reproductive isolation between sympatric races of pea aphids. Gene flow restriction and habitat choice. Evolution 53, 1446-1457.

Via, S., Bouck, A.C. \& Skillman, S. (2000) Reproductive isolation between divergent races of pea aphids on two hosts. II. Selection against migrants and hybrids in the parental environments. Evolution 54, 1626-1637.

Villada, E.S., González, E.G., López-Sesé, A.I., Castiel, A.F. \& Gómez-Guillamón, M.L. (2009) Hypersensitive response to Aphis gossypii Glover in melon genotypes carrying the Vat gene. Journal of Experimental Botany 60, 3269-3277.

Young, N.D., Debelle, F., Oldroyd, G.E.D., Geurts, R., Cannon, S.B., Udvardi, M.K., Benedito, V.A., Mayer, K.F. X., Gouzy, J., Schoof, H., Van de Peer, Y., Proost, S., Cook, D.R., Meyers, B.C., Spannagl, M., Cheung, F., De Mita, S., Krishnakumar, V., Gundlach, H., Zhou, S., Mudge, J., Bharti, A.K., Murray, J.D., Naoumkina, M.A., Rosen, B., Silverstein, K.A.T., Tang, H., Rombauts, S., Zhao, P.X., Zhou, P., Barbe, V., Bardou, P., Bechner, M., Bellec, A., Berger, A., Berges, H., Bidwell, S., Bisseling, T., Choisne, N., Couloux, A., Denny, R., Deshpande, S., Dai, X., Doyle, J.J., Dudez, A.-M., Farmer, A.D., Fouteau, S., Franken, C., Gibelin, C., Gish, J., Goldstein, S., Gonzalez, A.J., Green, P.J., Hallab, A., Hartog, M., Hua, A., Humphray, S.J., Jeong, D.-H., Jing, Y., Jocker, A., Kenton, S. M., Kim, D.-J., Klee, K., Lai, H., Lang, C., Lin, S., Macmil, S. L., Magdelenat, G., Matthews, L., McCorrison, J., Monaghan, E.L., Mun, J.-H., Najar, F.Z., Nicholson, C., Noirot, C., Bleness, M.O., Paule, C.R., Poulain, J., Prion, F., Qin, B., Qu, C., Retzel, E.F., Riddle, C., Sallet, E., Samain, S., Samson, N., Sanders, I., Saurat, O., Scarpelli, C., Schiex, T., Segurens, B., Severin, A.J., Sherrier, D.J., Shi, R., Sims, S., Singer, S.R., Sinharoy, S., Sterck, L., Viollet, A., Wang, B.B., Wang, K., Wang, M., Wang, X., Warfsmann, J., Weissenbach, J., White, D.D., White, J.D., Wiley, G.B., Wincker, P., Xing, Y., Yang, L., Yao, Z., Ying, F., Zhai, J., Zhou, L., Zuber, A., Denarie, J., Dixon, R.A., May, G.D., Schwartz, D.C., Rogers, J., Quetier, F., Town, C.D. \& Roe, B.A. (2011) The Medicago genome provides insight into the evolution of rhizobial symbioses. Nature 480, 520-524. 